\title{
Clinical Application of Technetium-99m-Labeled Red Blood Cells Subcutaneous Radionuclide Venography and Abdominal/Pelvic Hybrid SPECT/CT Imaging in Patients with Suspicion of Proximal Deep Vein Thrombosis
}

\author{
Chih-Ting Liu ${ }^{1}$, Hsiu-lan Chu ${ }^{1}$, I-Hsin Tsai ${ }^{1}$, Yi-Ling Chang ${ }^{2}$ and Chia-Yang Lin ${ }^{1 *}$ \\ ${ }^{1}$ Department of Nuclear Medicine, Kaohsiung Medical University Hospital, Kaohsiung, Taiwan \\ ${ }^{2}$ Chung Shan Medical University Hospital, Taichung, Taiwan
}

*Corresponding author: Chia-Yang Lin, Department of Nuclear Medicine, Kaohsiung Medical University Hospital, No.100, Tzyou $1^{\text {st }}$ Road Kaohsiung 807, Taiwan, Tel: 886-7-3121101; Fax: 886-7-3117521; E-mail: chiayanglin@gmail.com

Received date: May 10, 2016; Accepted date: August 04, 2016; Published date: August 09, 2016

Copyright: @ 2016 Liu CT, et al. This is an open-access article distributed under the terms of the Creative Commons Attribution License, which permits unrestricted use, distribution, and reproduction in any medium, provided the original author and source are credited.

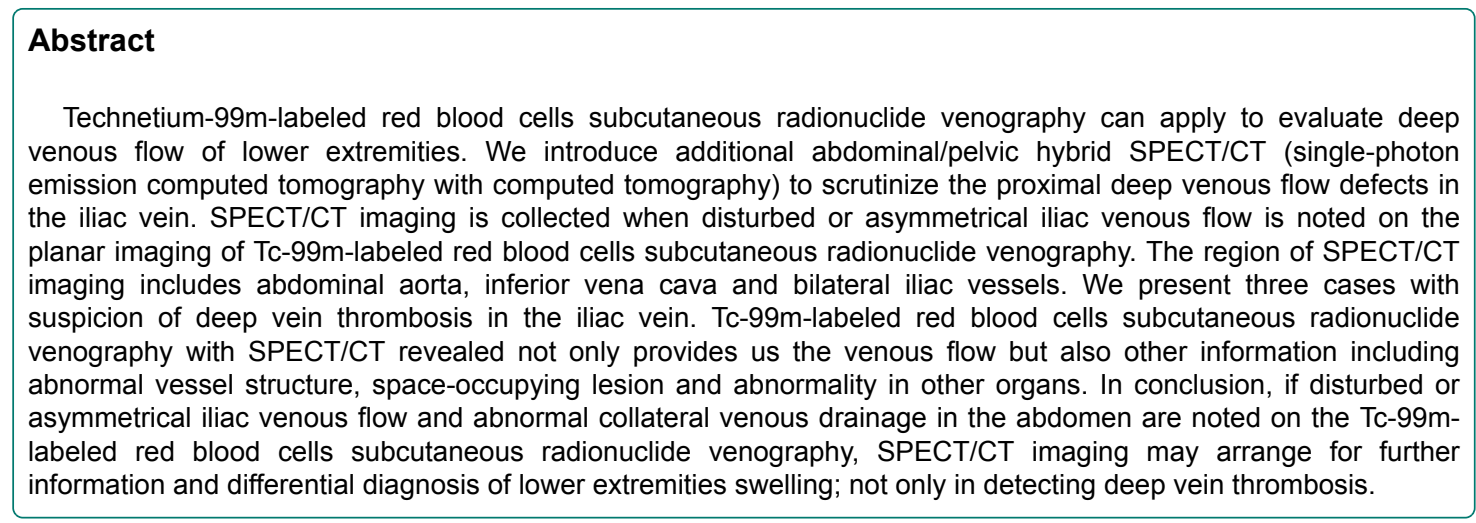

Keywords: Tc-99m-labeled red blood cells subcutaneous radionuclide venography; Single-photon emission computed tomography with computed tomography (SPECT/CT); Deep vein thrombosis

\section{Introduction}

There are many methods to complete radionuclide venography in the nuclear medicine department, including Technetium-99m MAA (Tc-99m macro aggregated albumin) radionuclide venography and Technetium-99m-labeled red blood cells subcutaneous radionuclide venography. Tc-99m-labeled red blood cells subcutaneous radionuclide venography using Tc-99m in vivo tagged red blood cells is an alternative procedure for conventional intravenous radionuclide venography $[1,2]$. In our hospital, we use in vivo labeling method. First of all, we inject stannous pyrophosphate with normal saline through intravenous route. About $15 \mathrm{~min}$ later, the patient is placed on the examination table in the supine position and we inject the dose of 6 $\mathrm{mCi}$ Tc-99m pertechnetate per limb via subcutaneous route between big toe and second toe on bilateral dorsum of foot (Figure 1A). By reduction of stannous pyrophosphate, Tc-99m can bind to red blood cells.

The early deep venous blood flow image is collected by means of tourniquets for compression of superficial venous drainage (Figure 1B). Half-body image from bilateral foot to dome of liver is acquired with low energy high-resolution collimator and at acquisition speeds of $18 \mathrm{~cm} / \mathrm{min}$. The injection site was not included due to scattering artifact. The blood-pool half-body image is collected without tourniquets. Image is acquired at acquisition speeds of $20 \mathrm{~cm} / \mathrm{min}$.
Single-photon emission computed tomography with computed tomography (SPECT/CT) imaging is arranged when proximal deep venous flow defects in the iliac vein are noted. The region of SPECT/CT imaging includes abdominal aorta, inferior vena cava and bilateral iliac vessels. The SPECT images setting are 32 projection angles and 30 second/angle. CT protocol consists of 1-mm-thick slices with tube voltage of $120 \mathrm{kV}$ and tube current setting of $30 \mathrm{~mA}$.

SPECT is reconstructed using iterative methods with a matrix size of $64 \times 64$. This reconstruction of CT is done with iterative reconstruction algorithms. We analyze the functional data from SPECT imaging with anatomical localization from CT imaging.

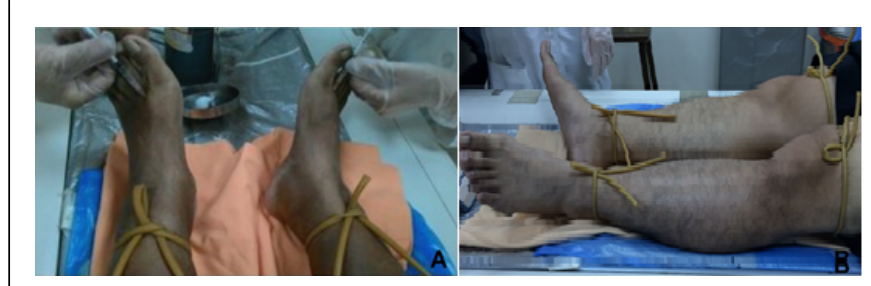

Figure 1: (A) Tc-99m pertechnetate injection via subcutaneous route between big toe and second toe on bilateral dorsum of foot. (B) Early deep venous blood flow image is collected by means of tourniquets for compression of superficial venous drainage. 
Citation: Liu CT, Chu HL, Tsai IH, Chang YL, Lin CY (2016) Clinical Application of Technetium-99m-Labeled Red Blood Cells Subcutaneous Radionuclide Venography and Abdominal/Pelvic Hybrid SPECT/CT Imaging in Patients with Suspicion of Proximal Deep Vein Thrombosis. J Nucl Med Radiat Ther 7: 295. doi:10.4172/2155-9619.1000295

Page 2 of 4

\section{Case Report}

\section{Case 1}

A 61-year-old male patient with past histories of diabetes mellitus, hypertension, old stroke without sequelae. One-day history of swelling in his right lower extremity. Tc-99m labeled red blood cells subcutaneous radionuclide venography was performed under suspicion of deep vein thrombosis. Irregular and interruption of deep venous flow were noted in the right lower extremity under tourniquets use. After tourniquets were removed, collateral vessels were noted in the right lower extremity (Figure 2). Lower extremity venous ultrasonography images proved deep vein thrombosis in the right femoral vein and knee (Figure 3). SPECT/CT imaging was performed for disturbed venous flow in the right iliac vein and revealed iliac venous engorgement with a hypodense lesion in the right iliac vein (Figure 4).

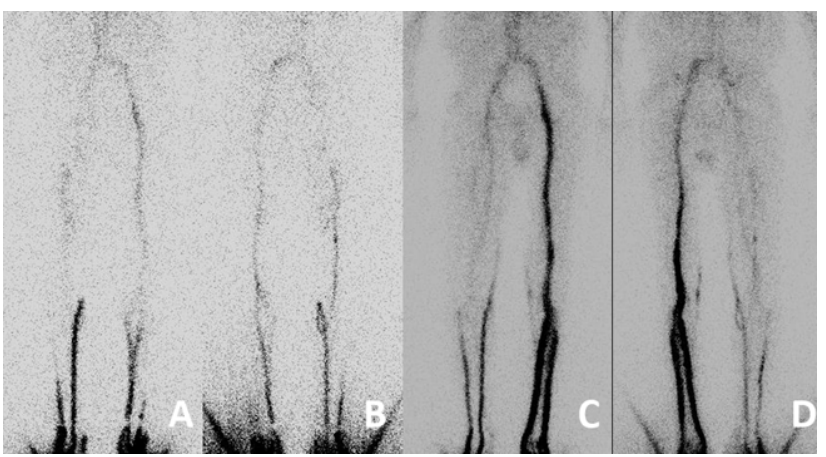

Figure 2: Tc-99m-labeled red blood cells subcutaneous radionuclide venography revealed irregular and interruption of deep venous flow in the right lower extremity under tourniquets use (A: anterior view; B: posterior view); after tourniquets were removed, collateral vessels were noted in the right lower extremity (C: anterior view; D: posterior view).

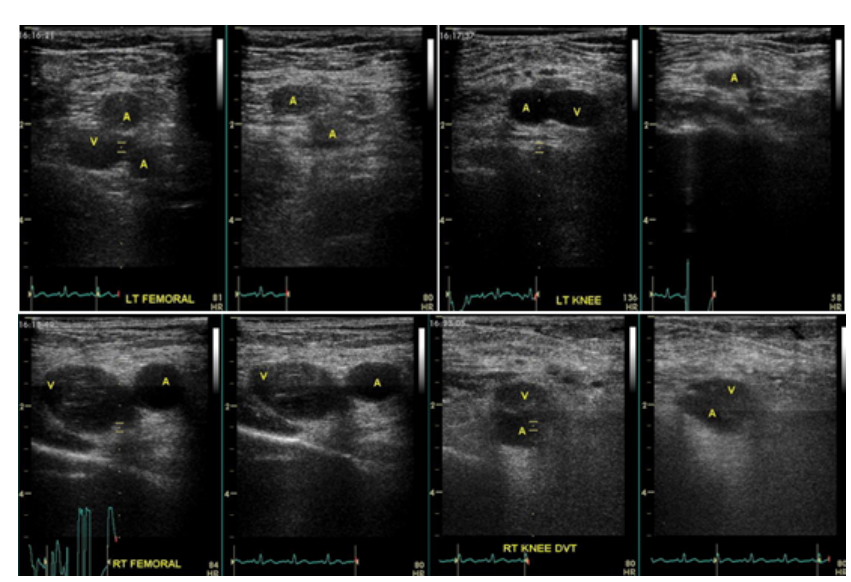

Figure 3: No evidence of deep vein thrombosis in the left femoral vein and left knee (A: artery and V: vein; upper row). Deep vein thrombosis in the right femoral vein and knee (A: artery and V: vein; lower row).

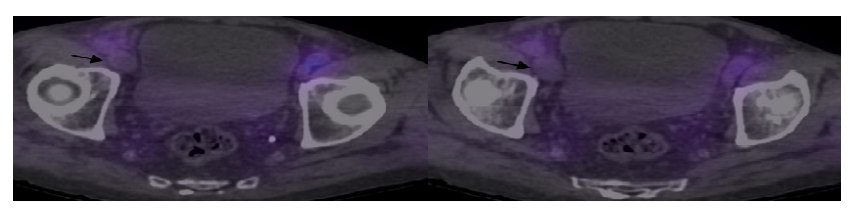

Figure 4: SPECT/CT imaging revealed iliac venous engorgement with a hypodense lesion in the right iliac vein (black arrow).

\section{Case 2}

A 33-year-old female patient with a past history of thalassemia. She complained of swelling with local heat in her left lower extremity for 4 days after delivery of her child. Tc-99m-labeled red blood cells subcutaneous radionuclide venography and imaging revealed disturbed deep venous flow in the left iliac vein, absent deep venous flow in the femoral vein with visualization of great saphenous vein (arrow) and abnormal collateral venous drainage (arrowhead) in the abdomen (Figure 5).

SPECT/CT imaging revealed left iliac venous engorgement and decreased radioactivity (Figure 6). Contrast computed tomography of abdomen revealed deep vein thrombosis in the inferior vena cava, the left common iliac, external iliac (Figure 7), internal iliac veins and the distal branches.

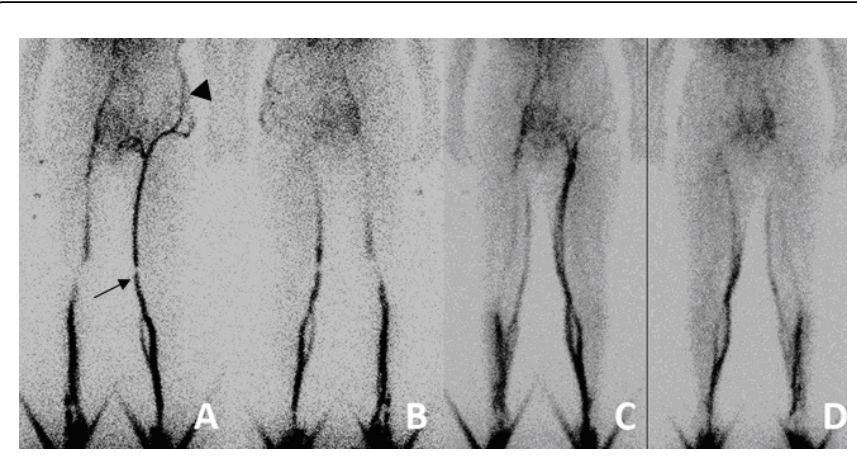

Figure 5: Tc-99m-labeled red blood cells subcutaneous radionuclide venography revealed disturbed deep venous flow in the left iliac vein, absent deep venous flow in the femoral vein with visualization of great saphenous vein (arrow) and abnormal collateral venous drainage (arrowhead) in the abdomen under tourniquets use (A: anterior view and B: posterior view). After tourniquets removal, similar pattern as images of tourniquets use (C: anterior view and D: posterior view).

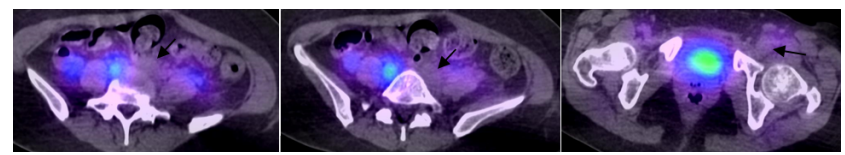

Figure 6: SPECT/CT imaging revealed left iliac vein engorgement and decreased radioactivity (black arrow). 
Citation: Liu CT, Chu HL, Tsai IH, Chang YL, Lin CY (2016) Clinical Application of Technetium-99m-Labeled Red Blood Cells Subcutaneous Radionuclide Venography and Abdominal/Pelvic Hybrid SPECT/CT Imaging in Patients with Suspicion of Proximal Deep Vein Thrombosis. J Nucl Med Radiat Ther 7: 295. doi:10.4172/2155-9619.1000295

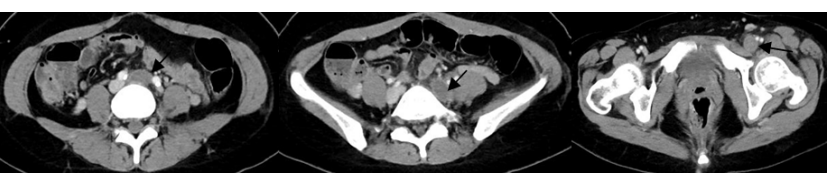

Figure 7: Contrast computed tomography of abdomen revealed deep vein thrombosis in the left common iliac and external iliac (black arrow).

\section{Case 3}

A 52-year-old male patient with past histories of papillary thyroid cancer, metastatic epidural tumor and L2 chordoma with surgical treatment. Tc-99m-labeled red blood cells subcutaneous radionuclide venography was performed for left lower extremity swelling. Planar imaging revealed disturbed venous flow in the iliac veins (arrow) with abnormal collateral venous drainage (arrowhead) in the abdomen (Figure 8). SPECT/CT imaging revealed displacement of inferior vena cava and suspicious tumor in the right paraspinal region (Figure 9). Magnetic resonance imaging (MRI) revealed worsening of metastasis in the T12-L3 vertebral bodies associated with paraspinal soft tissue invasion, tumor necrosis and inferior vena cava compression or direct invasion by tumor (Figure 10).

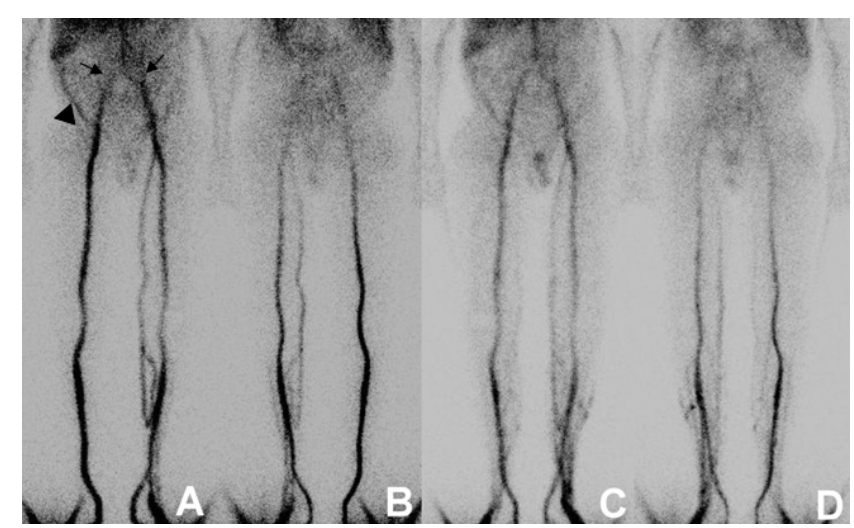

Figure 8: Tc-99m-labeled red blood cells subcutaneous radionuclide venography revealed disturbed venous flow in the iliac veins with abnormal collateral venous drainage in the abdomen (A and $\mathrm{B}$ under tourniquets use; $\mathrm{C}$ and $\mathrm{D}$ after tourniquets removal).

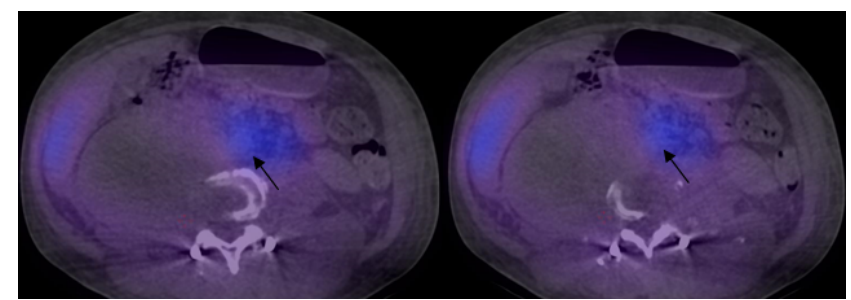

Figure 9: SPECT/CT imaging revealed displacement of inferior vena cava and suspicious tumor in the right paraspinal region (black arrow).

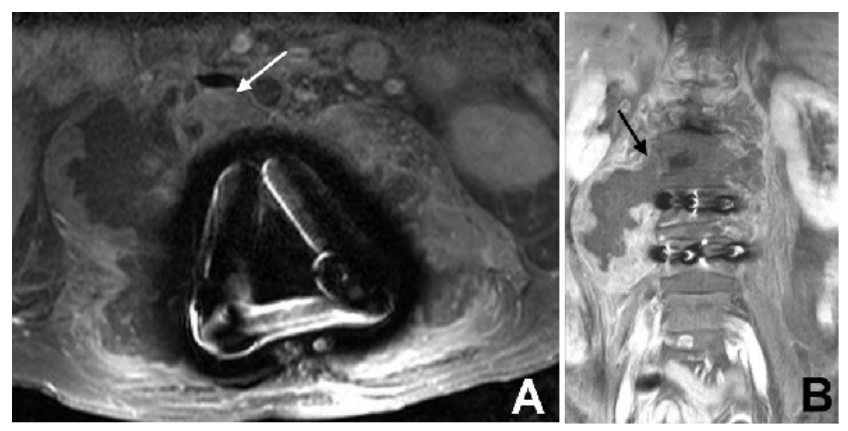

Figure 10: MRI imaging revealed worsening of metastasis in the T12-L3 vertebral bodies associated with paraspinal soft tissue invasion (B, black arrow), tumor necrosis and inferior vena cava compression or direct invasion by tumor (A, white arrow).

\section{Discussion}

Many patients are referred to our nuclear medicine department for suspicion of deep vein thrombosis. Intravenous radionuclide venography and radiographic contrast venography need intravenous administration. However, it is difficult to find a venous route from limb swelling. Thus, it is much easier to inject radiopharmaceutical via subcutaneous route.

Contrast venography has long been considered the reference test for the diagnosis of deep vein thrombosis. Due to other noninvasive tests, the need for contrast venography reduced. Contrast venography could not be performed in some patients due to contraindications or technical factors. Some possible risks of contrast venography included an allergic reaction to contrast material or a risk of injury to the kidneys in patients with impaired renal function. Compression ultrasonography has some limitations include not detect isolated thrombi in the iliac vein or that portion of the femoral vein within the adductor canal and pelvic neoplasms or abscesses may demonstrate isolated noncompressibility of the femoral vein when thrombosis is absent $[3,4]$. The benefit of ultrasonography is no radiation exposure. Due to above reasons, Tc-99m-labeled red blood cells subcutaneous radionuclide venography plays a role in this situation.

However, some patients may have an allergic reaction to stannous pyrophosphate. We should monitor patients' condition after stannous pyrophosphate injection. The radiation dose to the skin at the site of subcutaneous injection is approximately $0.02 \mathrm{~Gy} / \mathrm{mCi}$ according to previous study [2]. The dose-length product of SPECT/CT imaging in the abdomen/pelvis is less than that of contrast venography and contrast venography with higher effective dose.

Tc-99m-labeled red blood cells subcutaneous radionuclide venography with SPECT/CT provides us not only the venous flow in the iliac veins but also other information including abnormal vessel structure, space-occupying lesion and abnormality in other organs. May-Thurner syndrome is disease with abnormal vessel structure of the left common iliac vein stenosis secondary to compression of the left common iliac vein between the right common iliac artery and the underlying vertebral body [5]. If a patient with suspected May-Thurner syndrome on the SPECT/CT imaging, further imaging survey and treatments will be suggested. In our clinical experience, additional SPECT/CT imaging revealed psoas muscle abscess or deep vein 
Citation: $\quad$ Liu CT, Chu HL, Tsai IH, Chang YL, Lin CY (2016) Clinical Application of Technetium-99m-Labeled Red Blood Cells Subcutaneous Radionuclide Venography and Abdominal/Pelvic Hybrid SPECT/CT Imaging in Patients with Suspicion of Proximal Deep Vein Thrombosis. J Nucl Med Radiat Ther 7: 295. doi:10.4172/2155-9619.1000295

Page 4 of 4

thrombosis with hydroureter in a patient with a past history of colon cancer. These information may assist physicians in clinical treatment or assess the possible recurrence of colon cancer. Thus, Tc-99m-labeled red blood cells subcutaneous radionuclide venography with SPECT/CT provides the differential diagnosis of swelling in the lower extremities.

In conclusion, Tc-99m-labeled red blood cells subcutaneous radionuclide venography is a convenient method to evaluate the deep venous flow when patients have limb swelling. If disturbed or asymmetrical iliac venous flow and abnormal collateral venous drainage in the abdomen are noted on the Tc-99m-labeled red blood cells subcutaneous radionuclide venography, SPECT/CT imaging may arrange for further information such as abdominal and pelvic mass lesions [6]. Compression ultrasonography and Tc-99m-labeled red blood cells subcutaneous radionuclide venography with SPECT/CT imaging might play a complementary role in detecting deep vein thrombosis.

\section{Conflict of Interest}

We declare that we have no conflicts of interest.

\section{References}

1. Wu CC, Jong SB (1989) Radionuclide venography of lower limbs by subcutaneous injection: comparison with venography by intravenous injection. Ann Nucl Med 3: 125-133.

2. Yang DC, Ratani RS, Kalani J, Yu-Wen JC, Ying-Fong H, et al. (2001) Subcutaneous whole-body radionuclide venography using Tc-99m in vivo tagged red blood cells. Clin Nucl Med 26: 491-494.

3. Pinson AG, Becker DM, Philbrick JT, Parekh JS (1991) Technetium-99m$\mathrm{RBC}$ venography in the diagnosis of deep venous thrombosis of the lower extremity: a systematic review of the literature. J Nucl Med 32: 2324-2328.

4. Grant BJ (2014) Diagnosis of suspected deep vein thrombosis of the lower extremity. Uptodate.

5. Vijayalakshmi IB, Setty HS, Narasimhan C (2015) Unusual cases of rightsided and left-sided May-Thurner syndrome. Cardiol Young 25: 797-799.

6. Hsu CC, Chen YW, Chuang YW, Huang YF (2003) The Value of HalfBody Technetium-99m-Labeled Red Blood Cells Subcutaneous Radionuclide Venography in Differential Diagnosis of Lower Extremities Edema. Ann Nucl Med Sci 16: 47-51. 